\title{
Biregular cages of girth five
}

\author{
M. Abreu ${ }^{1 *} \quad$ G. Araujo-Pardo ${ }^{2 *} \quad$ C. Balbuena ${ }^{3 *}$ \\ D. Labbate ${ }^{1 *} \quad$ G. López-Chávez ${ }^{2}$ * \\ ${ }^{1}$ Dipartimento di Matematica, Informatica ed Economia \\ Università degli Studi della Basilicata, \\ Viale dell'Ateneo Lucano, I-85100 Potenza, Italy \\ marien.abreu@unibas.it; domenico.labbate@unibas.it \\ ${ }^{2}$ Instituto de Matemáticas \\ Universidad Nacional Autónoma de México \\ Ciudad Universitaria, México D.F. 04510, México. \\ garaujo@math.unam.mx; gloria04@gmail.com \\ 3 Departament de Matemàtica Aplicada III \\ Universitat Politècnica de Catalunya \\ Campus Nord, Edifici C2, C/ Jordi Girona 1 i 3 \\ E-08034 Barcelona, Spain. \\ m. camino.balbuena@upc.edu
}

Submitted: Aug 1, 2012; Accepted: Mar 25, 2013; Published: Mar 31, 2013

Mathematics Subject Classifications: 05C35

\begin{abstract}
Let $2 \leqslant r<m$ and $g$ be positive integers. An $(\{r, m\} ; g)$-graph (or biregular graph) is a graph with degree set $\{r, m\}$ and girth $g$, and an $(\{r, m\} ; g)$-cage (or biregular cage) is an $(\{r, m\} ; g)$-graph of minimum order $n(\{r, m\} ; g)$. If $m=r+1$, an $(\{r, m\} ; g)$-cage is said to be a semiregular cage.

In this paper we generalize the reduction and graph amalgam operations from [M. Abreu, G. Araujo-Pardo, C. Balbuena, D. Labbate. Families of Small Regular Graphs of Girth 5. Discrete Math. 312(18) (2012) 2832-2842] on the incidence graphs of an affine and a biaffine plane obtaining two new infinite families of biregular cages and two new semiregular cages. The constructed new families are $(\{r, 2 r-3\} ; 5)$-cages for all $r=q+1$ with $q$ a prime power, and $(\{r, 2 r-5\} ; 5)$-cages for all $r=q+1$ with $q$ a prime. The new semiregular cages are constructed for $r=5$ and 6 with 31 and 43 vertices respectively.
\end{abstract}

Keywords: biregular, cage, girth.

* Research supported by the Ministerio de Educación y Ciencia, Spain, and the European Regional Development Fund (ERDF) under project MTM2011-28800-C02-02, CONACyT-México under projects 166306 and 178395 and PAPIIT-México under project IN101912. 


\section{Introduction}

All graphs considered are finite, undirected and simple (without loops or multiple edges). For definitions and notations not explicitly stated the reader may refer to [15], [13] and [16].

Let $G$ be a graph with vertex set $V=V(G)$ and edge set $E=E(G)$. The girth of a graph $G$ is the length $g=g(G)$ of a shortest cycle. The degree of a vertex $v \in V$ is the number of vertices adjacent to $v$. A graph is called $r$-regular if all its vertices have the same degree $r$. A $(r, g)$-graph is a $r$-regular graph of girth $g$ and a $(r, g)$-cage is a $(r, g)$-graph with the smallest possible number of vertices. Cages have been intensely studied since they were introduced by Tutte [31] in 1947. Erdös and Sachs [20] proved the existence of a $(r, g)$-graph for any value of $r$ and $g$. Biggs is the author of an impressive report on distinct methods for constructing cubic cages [14]. More details about constructions of cages can be found in the surveys by Wong [32], by Holton and Sheehan [27, Chapter 6], or the recent one by Exoo and Jajcay [21].

The cages theory has been generalized in many ways, one such is as follows: if $D=$ $\left\{a_{1}, \ldots, a_{k}\right\}$ is a set of positive integers with $2 \leqslant a_{1}<a_{2}<\ldots<a_{k}$ then a $(D ; g)$-graph is a graph with degree set $D$ and girth $g$ and a $(D ; g)$-cage is a $(D ; g)$-graph with minimum order $n(D ; g)=n\left(a_{1}, \ldots, a_{k} ; g\right)$. It is obvious that the $(r ; g)$-cage is a special case of the $(D ; g)$-cage when $D=\{r\}$.

Few values of $n(D ; g)$ are known. In particular, Kapoor et al. [28] proved that $n(D ; 3)=1+a_{k}$. Moreover, the following lower bound for $n(D ; g)$ was given by Downs et al. [18]:

$$
n(D ; g) \geqslant \begin{cases}1+\sum_{i=1}^{t} a_{k}\left(a_{1}-1\right)^{i-1} & \text { if } g=2 t+1 \\ 1+\sum_{i=1}^{t-1} a_{k}\left(a_{1}-1\right)^{i-1}+\left(a_{1}-1\right)^{t-1} & \text { if } g=2 t .\end{cases}
$$

A biregular $(\{r, m\} ; g)$-graph is a $(D ; g)$-graph with degree set $D=\{r, m\}$ and girth $g$ and a bi-regular $(\{r, m\} ; g)$-cage is an $(\{r, m\} ; g)$-graph of smallest possible order. Note that bouquets of $r$ cycles of length $g$ are $(\{2,2 r\} ; g)$-cages and the complete bipartite graphs $K_{r, m}$ are $(\{r, m\} ; 4)$-cages. If $m=r+1$, an $(\{r, m\} ; g)$-cage is said to be a semiregular cage.

The existence of biregular $(\{r, m\} ; g)$-graphs has been proved by Chartrand, Gould, and Kapoor in [17] for all $2 \leqslant r<m$ and $g \geqslant 3$ (also proved by Füredi et al. in [23]). On the other hand, several contructions of biregular $(\{r, m\} ; g)$-cages have been achieved for different values of $r, m$ and $g$. In particular, Chartrand et al. proved in [17] that $n(\{r, m\} ; 4)=r+m$, for $2 \leqslant r<m$, and $g=4$ and they also proved in [17] that $n(\{2, m\} ; g)$ attains the lower bound (1). Furthermore, Yuansheng and Liang in [33] proved that $n(\{r, m\} ; 6) \geqslant 2(r m-m+1)$ for any $2 \leqslant r<m$; that $n(\{r, m\} ; 6)=$ $2(r m-m+1)$ for $g=6$ and $r<m$ when $2 \leqslant r \leqslant 5$ or $r \geqslant 2$ and $m-1$ a prime power; and they conjectured that $n(\{r, m\} ; 6)=2(r m-m+1)$, for any $2 \leqslant r<m$. 
In this paper, we focus our attention on biregular graphs of girth exactly five, where $n(\{r, m\} ; 5)=r m+1$. Some known results in this case, Downs et al. [18] have shown that $n(\{3 ; m\} ; 5)=3 m+1$, for any $m \geqslant 4$, Hanson et al. [24] have shown that $n(\{4 ; m\} ; 5)=$ $4 m+1$, for any integer $m \geqslant 5$, and Araujo-Pardo et al. in [7] proved several results in this context. Moreover, it is worth to note that Yuansheng and Liang in [33], claim to have proved that $n(\{5, m\} ; 5)=5 m+1$ for $m \geqslant 6$ in an unpublished manuscript. In Table 1 , we summarize these and other known results on the exact values of $n(\{r, m\} ; g)$, for $g \geqslant 5$, together with the results obtained in this paper which we now proceed to describe.

\begin{tabular}{|c|c|c|c|c|c|c|c|}
\hline$r$ & $m$ & $g=5$ & $g=6$ & $g=7$ & $g=8$ & $g=9$ & $g=11$ \\
\hline$r=3$ & $m \geqslant 4$ & $\begin{array}{c}3 m+1 \\
{[18]}\end{array}$ & $\begin{array}{l}4 m+2 \\
{[24,33]}\end{array}$ & $\begin{array}{c}7 m+1 \\
{[18]}\end{array}$ & $\begin{array}{c}\frac{25 m}{3}+5 \\
m \stackrel{3}{=} 3 k[7]\end{array}$ & \multirow{2}{*}{$\begin{array}{c}15 m+1 \\
m \geqslant 6 \\
{[18]}\end{array}$} & \multirow{2}{*}{$\begin{array}{c}31 m+1 \\
m=4 k \\
{[7]}\end{array}$} \\
\hline & & & & & $\begin{array}{c}9 m+3 \\
m=4,5,7[10]\end{array}$ & & \\
\hline$r=4$ & $m \geqslant 5$ & $\begin{array}{c}4 m+1 \\
{[24]}\end{array}$ & $\begin{array}{c}6 m+2 \\
{[33]}\end{array}$ & $\begin{array}{c}13 m+1 \\
m=6 k \\
{[7]}\end{array}$ & & & $\begin{array}{c}121 m+1 \\
m=6 k \\
{[7]}\end{array}$ \\
\hline \multirow{4}{*}{$\begin{array}{l}r \geqslant 5 \\
r=p^{h}+1 \\
p \text { prime }\end{array}$} & $m=2 k(r-1)$ & $\begin{array}{c}1+r m \\
{[7]}\end{array}$ & & \multirow[t]{5}{*}{$\begin{array}{c}1+m\left(r^{2}-r+1\right) \\
{[7]}\end{array}$} & & & \multirow[t]{5}{*}{$1+m \frac{(r-1)^{5}-1}{r-2}$} \\
\hline & $m=k(r-1)+1$ & & $\begin{array}{c}2(r m-m+1) \\
{[6,33]}\end{array}$ & & & & \\
\hline & $m=k r$ & & {$[7]$} & & & & \\
\hline & $m=2 r-3$ & $(*)$ & & & & & \\
\hline $\begin{array}{l}h=1 \\
p \geqslant 7\end{array}$ & $m=2 r-5$ & $(*)$ & & & & & \\
\hline
\end{tabular}

Table 1: Exact values of $n(\{r, m\} ; g)$. The symbol $\left(^{*}\right)$ means results obtained in this paper.

We generalize the reduction and graph amalgam operations from [1] on the incidence graphs $A_{q}$ of an affine and $B_{q}$ of a biaffine plane (elliptic semiplane of type $\mathcal{C}$ ) obtaining two new infinite families of biregular $(\{r, m\} ; 5)$-cages and two new semiregular cages of girth 5 . The constructed families are $(\{r, 2 r-3\} ; 5)$-cages for all $r=q+1$ with $q$ a prime power, and $(\{r, 2 r-5\} ; 5)$-cages for all $r=q+1$ with $q$ a prime. The new semiregular cages are constructed for $r=5$ and 6 with 31 and 43 vertices respectively.

The paper is organized as follows: the graphs $A_{q}$ and $B_{q}$ are presented in Section 2, with a labelling which will be necessary for the construction of biregular cages. In Section 3 , we construct previously unknown $(\{r, 2 r-3\} ; 5)$-cages, for a prime power $q \geqslant 2$ and the integer $r=q+1$, in Theorem 3.3, adding edges to the graph $A_{q}$. Moreover if $r-1$ is even, we exhibit $r$ non-isomorphic such $(\{r, 2 r-3\} ; 5)$-cages in Theorem 3.5. In particular, we find a $(\{4,5\} ; 5)$-semiregular cage with 21 vertices. In Section 4 we slightly generalize reduction and amalgam operations, described in [1] and [22], that, performed on the bipartite graph $B_{q}$, will allow us to construct new $(\{r, 2 r-5\} ; 5)$-cages, for $r=q+1$ with $q \geqslant 7$ prime, in Section 5, Theorems 5.10 and 5.11. Finally, in Section 6 we construct two new semiregular cages, namely a $(\{5,6\} ; 5)$-cage with 31 vertices and a $(\{6,7\} ; 5)$ cage with 43 vertices. Note that the latter is a sporadic example in which we adapt and slightly generalize the techniques that we have used in Section 4. 


\section{Preliminaries}

Let $q=p^{n} \geqslant 2$ be a prime power and $\alpha$ a primitive $(q-1)^{t h}$-root of unity. Consider the finite field $G F(q)=\left\{0,1, \alpha, \alpha^{2}, \ldots, \alpha^{q-2}\right\}$ and denote $G F^{*}(q)=G F(q) \backslash\{0\}$.

The graphs constructed in this paper arise from the (bipartite) incidence graph $B_{q}$ of an elliptic semiplane of type $C$ (cf. $[19,5,22]$ ) together with the (bipartite) incidence graph $A_{q}$ of the affine plane of order $q$. We fix a labelling on their vertices which will be central for our constructions since it allows us to keep track of the properties (such as regularity and girth) of the graphs obtained from $B_{q}$ and $A_{q}$ applying some operations such as reductions and amalgams (cf. Sections 3,4$)$.

Definition 2.1. Let $q \geqslant 2$ be a prime power, and consider the finite field $G F(q)$.

(i) Let $B_{q}$ be a bipartite graph with vertex set $\left(V_{0}, V_{1}\right)$ where $V_{r}=G F(q) \times G F(q)$, $r=0,1 ;$ and the edge set defined as follows:

$$
(x, y)_{0} \in V_{0} \text { adjacent to }(m, b)_{1} \in V_{1} \text { if and only if } y=m x+b .
$$

(ii) Let $A_{q}$ be the graph obtained from $B_{q}$ by adding the following set $L_{q}:=\left\{(q, x)_{1} \mid x \in\right.$ $G F(q)\}$ of $q$ vertices and the set $E_{q}:=\left\{u v \mid u:=(q, x)_{1}, v:=(x, y)_{0}\right.$ and $x, y \in$ $G F(q)\}$ of $q^{2}$ edges.

The graph $B_{q}$ is also known as the incidence graph of the biaffine plane [25] and the graph $A_{q}$ is the incidence graph of an affine plane of order $q$. The graph $B_{q}$ has been used in the problem of finding extremal graphs without short cycles (cf. e.g. [1, 3, 4, 8, 9, 11, 30]).

The following properties of the graph $B_{q}$ are well known (see $[1,25,30]$ ) and they will be fundamental throughout the paper.

Proposition 2.2. Let $B_{q}$ be the (bipartite) incidence graph defined above. Let $P_{x}=$ $\left\{(x, y)_{0} \mid y \in G F(q)\right\}$, for $x \in G F(q)$, and $L_{m}=\left\{(m, b)_{1} \mid b \in G F(q)\right\}$, for $m \in G F(q)$. Then the graph $B_{q}$ has the following properties:

(i) it is q-regular, vertex transitive, of order $2 q^{2}$ and has girth 6 for $q \geqslant 3$;

(ii) it admits a partition $V_{0}=\bigcup_{x \in G F(q)} P_{x}$ and $V_{1}=\bigcup_{m \in G F(q)} L_{m}$ of its vertex set;

(iii) each block $P_{x}$ is connected to each block $L_{m}$ by a perfect matching, for $x, m \in G F(q)$;

(iv) each vertex in $P_{0}$ and $L_{0}$ is connected straight to all its neighbours in $B_{q}$, meaning that $N\left((0, y)_{0}\right)=\left\{(i, y)_{1} \mid i \in G F(q)\right\}$ and $N\left((0, b)_{1}\right)=\left\{(j, b)_{0} \mid j \in G F(q)\right\}$;

(v) the other matchings between $P_{x}$ and $L_{m}$ are twisted and the rule is defined algebraically in $G F(q)$ according to (2).

For further information regarding these properties and for constructions of the adjacency matrix of $B_{q}$ as a block $(0,1)$-matrix please refer to $[2,5,12]$. 


\section{Construction of a family of $(\{r, 2 r-3\} ; 5)$-cages.}

In this section, for a prime power $q \geqslant 2$ and the integer $r=q+1$, we construct previously unknown $(\{r, 2 r-3\} ; 5)$-cages, adding edges to the graph $A_{q}$ presented in Section 2 .

Let $q \geqslant 2$ be a prime power and let $r=q+1$. We define $R_{q}$ to be the graph with $V\left(R_{q}\right):=V\left(A_{q}\right)$ and $E\left(R_{q}\right):=E\left(A_{q}\right) \cup D$ where $D=\left\{(m, 0)_{1}(m, b)_{1} \mid b \in G F^{*}(q)\right.$ and $m \in G F(q) \cup\{q\}\}$.

Theorem 3.3. Let $q \geqslant 2$ be a prime power and let $r=q+1$. Then the graph $R_{q}$ is a $(\{r, 2 r-3\} ; 5)$-cage satisfying Downs' bound, i.e. $n(\{r, 2 r-3\} ; 5)=r(2 r-3)+1$.

Proof. The vertices $M:=\left\{(m, 0)_{1} \mid m \in G F(q) \cup\{q\}\right\} \subset V\left(R_{q}\right)$ have degree $q+(q-1)=$ $2 q-1=2 r-3$, and the remaining vertices of $R_{q}$ have degree $q+1=r$.

By construction $B_{q} \subset A_{q} \subset R_{q}$. Let $C$ be a cycle in $R_{q}$. If the edges of $C$ are totally contained in $A_{q}$ then the length of $C$ is at least six, since $A_{q}$ is the incidence graph of an affine plane. Otherwise, $C$ contains at least an edge $e=x y \in D$, where $x, y \in L_{m}$ and $m \in G F(q) \cup\{q\}$. Then it follows from the bipartition and girth of $A_{q}$ that the distance $d_{A_{q}}(x, y)=4$. Thus the length of $C$ in this case is at least five and exactly five if $C$ contains exactly one edge of $D$. Hence, the graph $R_{q}$ has girth 5 since $C=(q, 0)_{1}(q, 1)_{1}(1,0)_{0}(0,0)_{1}(0,0)_{0}(q, 0)_{1}$ where $(q, 0)_{1}(q, 1)_{1}$ is the only edge of $C$ in $D$.

Finally, $\left|V\left(R_{q}\right)\right|=\left|V\left(A_{q}\right)\right|=2 q^{2}+q=2 r^{2}-3 r+1=r(2 r-3)+1$.

Corollary 3.4. The graph $R_{q}$ is a semi-regular cage if and only if $r=4$.

Proof. It follows immediately since $2 r-3=r+1$ if and only if $r=4$.

Note that an isomorphic graph to $R_{3}$ has been found also in [24].

In a similar way, for $q$ even, we construct a family of non-isomorphic $(\{r, 2 r-3\} ; 5)$-cages.

Let $q$ be an even prime power and let $D_{m}:=\left\{(m, 0)_{1}(m, b)_{1} \mid b \in G F(q)\right\}$ and $F_{m}:=$ $\left\{(m, 0)_{1}(m, 1)_{1}\right\} \cup\left\{\left(m, \alpha^{i}\right)_{1}\left(m, \alpha^{i+1}\right)_{1} \mid 1 \leqslant i \leqslant q-3\right.$, $i$ odd $\}$, for $m \in G F(q) \cup\{q\}$. Then $D_{m} \cong K_{1, q-1}$ is a star with vertex set $L_{m}$ and $F_{m}$ is a matching between the vertices of $L_{m}$.

Let $0 \leqslant t \leqslant q-1$ and let $I_{t}=\left\{\alpha^{q-t-1}, \ldots, \alpha^{q-2}\right\}$ be a set of indexes. We define $G_{t}$ to be the graph with $V\left(G_{t}\right):=V\left(A_{q}\right)$ and $E\left(G_{t}\right):=E\left(A_{q}\right) \cup F_{t} \cup D_{t}$, where

$$
F_{t}:=\bigcup_{m \in G F(q) \backslash I_{t}} F_{m} \text { and } D_{t}:=\bigcup_{m \in I_{t} \cup\{q\}} D_{m}
$$

Note that the graph $G_{t}$ is obtained from the graph $A_{q}$ adding $t+1$ stars and $q-t$ matchings within the sets $L_{m}$. In particular, for $t=0$ the index set $I_{t}=\emptyset$ and the only star added to $A_{q}$ is the one in $L_{q}$. Moreover, if we set $I_{q}:=G F(q)$, then we can say that the graph $G_{q}:=R_{q}$.

Theorem 3.5. Let $q=2^{s}$ be an even prime power, with $s>1$. Then there are at least $q+1$ non-isomorphic $(\{r, 2 r-3\} ; 5)$-cages. 
Proof. Let $G_{t}$ be the family of graphs defined as above, for $0 \leqslant t \leqslant q$. Reasoning as in Theorem 3.3, is follows that, for every $0 \leqslant t \leqslant q-1$, the graphs $G_{t}$ have girth 5, order $r(2 r-3)+1$ and are biregular with $t+1$ vertices of degree $2 r-3$. Thus, $G_{t}$ is a $(\{r, 2 r-3\} ; 5)$-cage for every $0 \leqslant t \leqslant q-1$. Moreover, $G_{i} \not G_{j}$, for $i, j \in G F(q) \cup\{q\}$ with $i \neq j$, since they have a different number of vertices of degree $2 r-3$.

\section{Operations on $B_{q}$}

In this section we slightly generalize reduction and amalgam operations, described in [1] and [22], that, performed on the bipartite graph $B_{q}$, will allow us to construct new $(\{r, 2 r-5\} ; 5)$-cages, for $r=q+1$ with $q \geqslant 7$ prime, in Section 5 .

\subsection{Reductions}

We describe two reduction operations on $B_{q}$ already introduced in [1]. The first one is exactly the same while the second one is slightly generalized.

Reduction 1[1] Remove vertices from $P_{0}$ and $L_{0}$.

Let $T \subseteq S \subseteq G F(q), S_{0}=\left\{(0, y)_{0} \mid y \in S\right\} \subseteq P_{0}, T_{0}=\left\{(0, b)_{1} \mid b \in T\right\} \subseteq L_{0}$ and $B_{q}(S, T)=B_{q}-S_{0}-T_{0}$.

Lemma 4.6. Let $T \subseteq S \subseteq G F(q)$. Then $B_{q}(S, T)$ is biregular with degrees $(q-1, q)$ of order $2 q^{2}-|S|-|T|$. Moreover, the vertices $(i, t)_{0} \in V_{0}$ and $(j, s)_{1} \in V_{1}$, for each $i, j \in G F(q)-\{0\}, s \in S$ and $t \in T$ are the only vertices of degree $q-1$ in $B_{q}(S, T)$, together with $(0, s)_{1} \in V_{1}$ for $s \in S-T$ if $T \subsetneq S$.

Proof. It is an immediate consequence of Proposition $2.2(i),(v)$.

Reduction 2 Remove blocks $P_{i}$ and $L_{j}$ from $B_{q}$ or from $B_{q}(S, T)$.

Let $u_{0}, u_{1}$ be non-negative integers such that $0 \leqslant u_{0} \leqslant u_{1}<q-1$. If $u_{i}>0$, let $U_{i}:=\left\{\alpha^{q-j} \in G F(q): j=2, \ldots, u_{i}+1\right\}$ be an index set, for $i=0,1$. Let $\mathcal{U}_{0}$ and $\mathcal{U}_{1}$ be sets of blocks of $B_{q}$ chosen as follows:

$$
\begin{array}{cll}
\mathcal{U}_{0}:=\left\{P_{x} \subset V_{0}: x \in U_{0}\right\} \text { if } 1 \leqslant u_{0} \leqslant q-1 & \text { or } & \mathcal{U}_{0}:=\emptyset \text { if } u_{0}=0 \\
\mathcal{U}_{1}:=\left\{L_{m} \subset V_{1}: m \in U_{1}\right\} \text { if } 1 \leqslant u_{1} \leqslant q-1 & \text { or } & \mathcal{U}_{1}:=\emptyset \text { if } u_{1}=0 .
\end{array}
$$

Then, we define $B_{q}\left(u_{0}, u_{1}\right):=B_{q}-\mathcal{U}_{0}-\mathcal{U}_{1}$ to be the graph obtained from $B_{q}$ by deleting the last $u_{0}$ blocks of $V_{0}$, and the last $u_{1}$ blocks of $V_{1}$. Analogously, we define $B_{q}\left(S, T, u_{0}, u_{1}\right):=$ $B_{q}-\left(S_{0} \cup T_{0}\right)-\left(\mathcal{U}_{0} \cup \mathcal{U}_{1}\right)$ to be the graph obtained from the graph $B_{q}(S, T)$ obtained with Reduction 1. Clearly, for $u_{0}=u_{1}=0, B_{q}(0,0)=B_{q}$ and $B_{q}(S, T, 0,0)=B_{q}(S, T)$.

Lemma 4.7. Let $u_{0}, u_{1}$ be non-negative integers, with $0 \leqslant u_{0} \leqslant u_{1}<q-1$. Then

(i) the graph $B_{q}\left(u_{0}, u_{1}\right)$ is a biregular graph with degrees $\left\{q-u_{0}, q-u_{1}\right\}$ of order $2 q^{2}-$ $q\left(u_{0}+u_{1}\right)$ if $u_{0} \neq u_{1}$; 
(ii) the graph $B_{q}\left(u_{0}, u_{1}\right)$ is a $\left(q-u_{0}\right)$-regular graph of order $2 q\left(q-u_{0}\right)$ if $u_{0}=u_{1}$;

(iii) the graph $B_{q}\left(S, T, u_{0}, u_{1}\right)$ has degrees $\left\{q-u_{0}, q-u_{1}, q-u_{0}-1, q-u_{1}-1\right\}$ and order $2 q^{2}-q\left(u_{0}+u_{1}\right)-|S|-|T|$. Moreover, the vertices $(i, t)_{0} \in V_{0}$ with $i \in G F(q)-U_{0}$ and $t \in T$ are the only vertices of degree $q-u_{1}-1$ in $B_{q}\left(S, T, u_{0}, u_{1}\right)$ and the vertices $(j, s)_{1} \in V_{1}$ with $j \in G F(q)-U_{1}$ and $s \in S$, together with the vertices $(0, s)_{1} \in V_{1}$, for $s \in S-T$ if $T \subsetneq S$, are the only vertices of degree $q-u_{0}-1$ in $B_{q}\left(S, T, u_{0}, u_{1}\right)$;

Proof. It is an immediate consequence of Proposition $2.2(i),(v)$ and Lemma 4.6.

\subsection{Amalgam}

In this section we describe an amalgam operation inspired by Jørgensen [29], Funk [22] and Abreu et al. [1], where regular bipartite graphs were transformed into (no longer bipartite) regular graphs of higher degree adding weighted edges with different weights on opposite sides of the bipartition.

Since we apply Reduction 1 before increasing the degree of $B_{q}$, we describe the amalgam operation performed on the reduced graph $B_{q}\left(S, T, u_{0}, u_{1}\right)$ for $0 \leqslant u_{0} \leqslant u_{1}<q-1$. The labelling for $B_{q}$ introduced in Section 2, will be essential, in the choice of the graphs used for the amalgam, to guarantee the biregularity and the girth 5 in the final graph.

Let $\Gamma_{1}$ and $\Gamma_{2}$ be two graphs of the same order and with the same labels on their vertices. In general, an amalgam of $\Gamma_{1}$ into $\Gamma_{2}$ is a graph obtained adding all the edges of $\Gamma_{1}$ to $\Gamma_{2}$.

Let $P_{i}$ and $L_{i}$ be defined as in Section 2. Consider the graph $B_{q}\left(S, T, u_{0}, u_{1}\right)$, for some $T \subseteq S \subseteq G F(q)$ and $0 \leqslant u_{0} \leqslant u_{1}<q-1$. Let $S_{0} \subseteq P_{0}, T_{0} \subseteq L_{0}$ as in Reduction 1 , and let $P_{0}^{\prime}:=P_{0}-S_{0}$ and $L_{0}^{\prime}:=L_{0}-T_{0}$ be the blocks in $B_{q}\left(S, T, u_{0}, u_{1}\right)$ of order $q-|S|$ and $q-|T|$, respectively.

Let $H_{i}, G_{i}$, for $i=0,1$, be graphs of girth at least 5 and order $q-|S|, q-|T|$ and $q$, respectively. To simplify notation in our results, we label $P_{i}$ and $L_{i}$ as in Section 2 , but assume that the labellings of $H_{0}, H_{1}, G_{0}$ and $G_{1}$ correspond to the second coordinates of $P_{0}^{\prime}, L_{0}^{\prime}, P_{i}$ and $L_{j}$ respectively for $i \in G F^{*}(q)-U_{0}$ and $j \in G F^{*}(q)-U_{1}$.

We define $B_{q}^{*}\left(S, T, u_{0}, u_{1}\right)$ to be the amalgam of $H_{0}$ into $P_{0}^{\prime}, H_{1}$ into $L_{0}^{\prime}, G_{0}$ into $P_{i}$, for $i \in G F^{*}(q)-U_{0}$, and $G_{1}$ into $L_{j}$, for $j \in G F^{*}(q)-U_{1}$. Note that $\left|V\left(B_{q}^{*}\left(S, T, u_{0}, u_{1}\right)\right)\right|=$ $\left|V\left(B_{q}\left(S, T, u_{0}, u_{1}\right)\right)\right|$.

The next lemma is immediate and it shows the behavior of the degree set of the graph $B_{q}^{*}\left(S, T, u_{0}, u_{1}\right)$.

Lemma 4.8. Let $G:=B_{q}^{*}\left(S, T, u_{0}, u_{1}\right)$. Then the degrees of the vertices of $G$ are:

1. $d_{G}\left((0, y)_{0}\right)=q-u_{1}+d_{H_{0}}(y)$,

2. $d_{G}\left((0, b)_{1}\right)=q-u_{0}+d_{H_{1}}(b)$ if $b \notin S-T$,

3. $d_{G}\left((0, b)_{1}\right)=q-u_{0}-1+d_{H_{1}}(b)$ if $b \in S-T$,

4. $d_{G}\left((x, y)_{0}\right)=q-u_{1}+d_{G_{0}}(y)$ if $y \notin T$, 
5. $d_{G}\left((x, y)_{0}\right)=q-u_{1}-1+d_{G_{0}}(y)$ if $y \in T$,

6. $d_{G}\left((m, b)_{1}\right)=q-u_{0}+d_{G_{1}}(b)$ if $b \notin S$,

7. $d_{G}\left((m, b)_{1}\right)=q-u_{0}-1+d_{G_{1}}(b)$ if $b \in S$,

Note that with the above mentioned labelling, the labels of $G_{0}, G_{1}$ are the elements of $G F(q)$ and the labels of $H_{0}, H_{1}$ are either the elements of $G F(q)$ itself or a proper subset according to $S, T$ being empty or not.

Let $M_{F}:=\{(u, v): u, v \in G F(q)$ and $u v \in E(F)\}$, for $F \in\left\{H_{0}, H_{1}, G_{0}, G_{1}\right\}$. For each $(u, v) \in M_{F}$, we define $\omega((u, v))= \pm(u-v) \in G F^{*}(q)$ to be its weight or Cayley Colour. We define $\Omega(F):=\left\{\omega((u, v)):(u, v) \in M_{F}\right\}$ to be the set of weights or set of Cayley Colours of $F$, for $F \in\left\{H_{0}, H_{1}, G_{0}, G_{1}\right\}$.

Note that $\Omega\left(F_{1}\right) \cap \Omega\left(F_{2}\right)=\emptyset$ implies that $M_{F_{1}} \cap M_{F_{2}}=\emptyset$, for $F_{1}, F_{2} \in\left\{H_{0}, H_{1}, G_{0}, G_{1}\right\}$ and $F_{1} \neq F_{2}$, but the converse is false.

The following lemma generalizes theorems [1, Theorem 5] and [22, Theorem 2.8].

Theorem 4.9. Let $T \subseteq S \subseteq G F(q)$ and let $0 \leqslant u_{0} \leqslant u_{1}<q-1$. Let $H_{0}, H_{1}, G_{0}$ and $G_{1}$ be defined as above and suppose that $M_{H_{0}} \cap M_{H_{1}}=\emptyset, M_{H_{0}} \cap M_{G_{1}}=\emptyset, M_{H_{1}} \cap M_{G_{0}}=\emptyset$ and $\Omega\left(G_{0}\right) \cap \Omega\left(G_{1}\right)=\emptyset$. Then the amalgam $B_{q}^{*}\left(S, T, u_{0}, u_{1}\right)$ has girth at least 5 and order $2 q^{2}-q\left(u_{0}+u_{1}\right)-|S|-|T|$.

Proof. Suppose first that $u_{0}=u_{1}=0$ and so $B_{q}^{*}(S, T)=B_{q}^{*}\left(S, T, u_{0}, u_{1}\right)$ (cf. Reduction 2).

Let $C$ be a shortest cycle in $B_{q}^{*}(S, T)$ and suppose, by contradiction, that $|C| \leqslant 4$. Therefore, $C=(x y z)$ or $C=(w x y z)$. Since $B_{q}$ has girth 6 and $H_{0}, H_{1}, G_{0}, G_{1}$ have girth at least 5, then $C$ cannot be completely contained in $B_{q}$ or in $H_{0}, H_{1}, G_{0}$ or $G_{1}$. Then, w.l.o.g. the path $x y z$ in $C$ is such that $x, y \in P_{i}$ and $z \in L_{m}$ for some $i, m \in G F(q)$. Since the edges between $P_{i}$ and $L_{m}$ form a matching, then $x z \notin E\left(B_{q}\right)$ and hence $x z \notin$ $E\left(B_{q}^{*}(S, T)\right)$. Thus $|C|>3$ and we can assume $|C|=4$ and $C=($ wxyz), with $x y z$ taken as before.

If $w \in P_{i}$, by the same argument, $w z \notin E\left(B_{q}^{*}(S, T)\right)$ and we have a contradiction. There are no edges between $P_{i}$ and $P_{j}$ in $B_{q}^{*}(S, T)$, so $w \notin P_{j}$ for $j \in G F(q)-\{i\}$, which implies that $w \in L_{n}$ for some $n \in G F(q)$. If $n \neq m$, we have a contradiction since there are no edges between $L_{m}$ and $L_{n}$ in $B_{q}^{*}(S, T)$. Therefore $x, y \in P_{i}$ and $w, z \in L_{m}$. Let $x=\left(i, a_{1}\right)_{0}, y=\left(i, a_{2}\right)_{0}, w=\left(m, b_{1}\right)_{1}$ and $z=\left(m, b_{2}\right)_{1}$ as in the labelling chosen in Section 2. Then $w x, y z \in E\left(B_{q}^{*}(S, T)\right)$ imply that $a_{1}=m \cdot i+b_{1}$ and $a_{2}=m \cdot i+b_{2}$, respectively.

If $m$ or $i$ are zero, i.e. if $x y \in H_{0}$ or $w z \in H_{1}$, then the above equations are satisfied if and only if $a_{1}=b_{1}$ and $a_{2}=b_{2}$, but this contradicts at least one of $M_{H_{0}} \cap M_{H_{1}}=\emptyset$, $M_{H_{0}} \cap M_{G_{1}}=\emptyset$ and $M_{H_{1}} \cap M_{G_{0}}=\emptyset$.

If $m$ and $i$ are both non-zero, i.e. if $x y \in G_{0}$ and $w z \in G_{1}$, then the above equations are satisfied if and only if $a_{1}-a_{2}=b_{1}-b_{2}$, implying that $\pm\left(a_{1}-a_{2}\right) \in \Omega\left(G_{0}\right)$ and $\pm\left(a_{1}-a_{2}\right)= \pm\left(b_{1}-b_{2}\right) \in \Omega\left(G_{1}\right)$ which contradicts $\Omega\left(G_{0}\right) \cap \Omega\left(G_{1}\right)=\emptyset$.

Hence $B_{q}^{*}(S, T)$ has girth at least five. Since $B_{q}^{*}\left(S, T, u_{0}, u_{1}\right)$ is a subgraph of $B_{q}^{*}(S, T)$, for $0 \leqslant u_{0} \leqslant u_{1}<q-1$, then also $B_{q}^{*}\left(S, T, u_{0}, u_{1}\right)$ ha girth five, completing the proof. 


\section{$5 \quad$ New $(\{r, 2 r-5\} ; 5)$-cages for $r \geqslant 8$.}

In this section we will construct a new family of $(\{r, 2 r-5\} ; 5)$-cages, for $r=q+1$ and $q \geqslant 7$ a prime, applying Reduction 1, 2 and Amalgam (cf. Theorem 4.9 and Lemma 4.8) to the graph $B_{q}$ as previously described.

Recall that every prime $q$ is either congruent to 1 or 3 modulo 4 , we will now treat these two cases separately, when $q=4 n+1$ or $q=4 n+3$. In each case we will specify the sets $S$ and $T$ to be deleted from $P_{0}$ and $L_{0}$, the integers $u_{0}$ and $u_{1}$ of number of blocks to be deleted, and the graphs $H_{0}, H_{1}, G_{0}$ and $G_{1}$ to be used for the amalgam into $B_{q}^{*}\left(S, T, u_{0}, u_{1}\right)$.

Since $q$ is a prime we can consider that $G F(q)$ coincides with $\mathbb{Z}_{q}$, and the addition operations are modulo $q$. We present the case $q \equiv 3 \bmod 4$ first, since the smallest case of the construction occurs for $q=7 \equiv 3 \bmod 4$. In what follows, recall that $D(F)$ denotes the degree set of a graph $F$.

\subsection{Construction for primes $q=4 n+3, n \geqslant 1$.}

Let $B_{q}^{*}\left(S, T, u_{0}, u_{1}\right)$ be the graph resulting from the following choice of its parameters:

\begin{tabular}{|l|c|c|l|}
\hline \multicolumn{5}{|c|}{$S=\left\{\frac{q+1}{4},-\frac{q+1}{4}\right\}=\left\{\frac{q+1}{4}, \frac{3 q-1}{4}\right\} ; \quad T=\emptyset ; \quad u_{0}=0 ; \quad u_{1}=1$} \\
\hline Graph & Vertices & Edges & Description \\
\hline$H_{0}$ & $\mathbb{Z}_{q}-\left\{\frac{q+1}{4}, \frac{3 q-1}{4}\right\}$ & $\left\{\left(j, j+\frac{q-1}{2}\right) \mid j \in \mathbb{Z}_{q}-\left\{\frac{q+1}{4}, \frac{3 q-1}{4}, \frac{3 q+3}{4}\right\}\right\} \cup\left\{\left(\frac{3 q+3}{4}, \frac{q-3}{4}\right)\right\}$ & $\begin{array}{l}(q-2) \text {-cycle } \\
\text { sums modulo } q\end{array}$ \\
\hline$G_{0}$ & $\mathbb{Z}_{q}$ & $\begin{array}{c}\left\{\left(j, j+\frac{q-1}{2}\right) \mid j \in \mathbb{Z}_{q}\right\} \\
\text { sums modulo } q\end{array}$ & $\begin{array}{l}q-\text { cycle } \\
\Omega\end{array}$ \\
\hline$H_{1} \cong G_{1}$ & $\mathbb{Z}_{q}$ & $\left.\left\{(0, j): j \in \mathbb{Z}_{q}^{*}-\left\{\frac{q-1}{2}, \frac{q+1}{2}\right\}\right\} \cup\left\{\left(\frac{q+1}{4}, \frac{q-1}{2}\right),\left(\frac{3 q-1}{4}, \frac{q+1}{2}\right)\right\}\right\}$ & $\Omega\left(H_{1}\right)=\Omega\left(G_{1}\right)=\mathbb{Z}_{q}^{*}-\left\{\frac{q-1}{2}\right\}$ \\
\hline
\end{tabular}

To illustrate the construction we present in Figure 1 the graph $B_{q}^{*}\left(S, T, u_{0}, u_{1}\right)$ without the edges from $B_{q}$, for $q=7$. Each line style represents a different weight (or Cayley Colour). As we will proved in the Theorem 5.10, this is an $(\{8,11\} ; 5)$-cage.

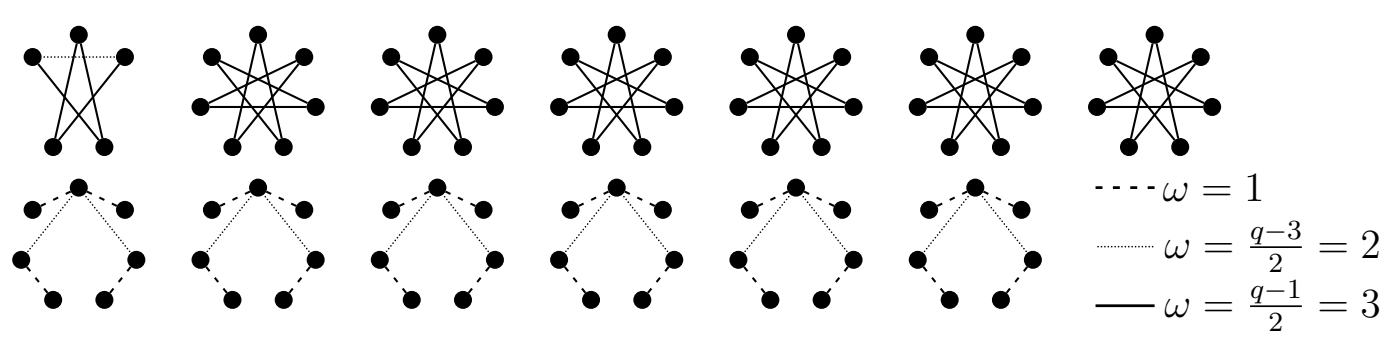

Figure 1: $B_{7}^{*}(S, T, 0,1)-E\left(B_{7}\right)$ with $S=\{3,5\}$ and $T=\emptyset$

Theorem 5.10. Let $q=4 n+3$ be a prime, for $n \geqslant 1$. Let $S, T, u_{0}, u_{1}, H_{0}, H_{1}, G_{0}$ and $G_{1}$ be defined as above. Then the amalgam graph $B_{q}^{*}\left(S, T, u_{0}, u_{1}\right)$ is an $(\{r, 2 r-5\} ; 5)$-cage of order $r(2 r-5)+1$, where $r=q+1$. 
Proof. From Lemma 4.8, the degree set $D\left(B_{q}^{*}\left(S, T, u_{0}, u_{1}\right)\right)=\{r, 2 r-5\}$, where $r=q+1$. In fact, all vertices in $V_{0} \cap B_{q}\left(S, T, u_{0}, u_{1}\right)$ have degree $q-1$. Moreover, $D\left(H_{0}\right)=D\left(G_{0}\right)=$ $\{2\}$ since $H_{0}$ and $G_{0}$ are cycles. Hence, all the vertices of $V_{0} \cap V\left(B_{q}^{*}\left(S, T, u_{0}, u_{1}\right)\right)$ have degree $q+1=r$. In $V_{1}$ we distinguish three subsets of vertices: $V_{1}^{\prime}:=\left\{(m, 0)_{1}: m \in \mathbb{Z}_{q}\right\}$, $V_{1}^{\prime \prime}:=\left\{(m, t)_{1}: m \in \mathbb{Z}_{q}, t \in \frac{q+1}{4}, \frac{3 q-1}{4}\right\}$ and $V_{1}^{\prime \prime \prime}:=\left\{(m, t)_{1}: t \in \mathbb{Z}_{q}^{*}-\left\{\frac{q+1}{4}, \frac{3 q-1}{4}\right\}\right\}$. The vertices in $V_{1}^{\prime \prime \prime}$ have degree $q$ in $B_{q}\left(S, T, u_{0}, u_{1}\right)$, and their degree is 1 in $H_{1}$ and $G_{1}$. Thus these vertices have degree $q+1=r$ in $B_{q}^{*}\left(S, T, u_{0}, u_{1}\right)$. The vertices of $V_{1}^{\prime \prime}$ have degree $q-1$ in $B_{q}\left(S, T, u_{0}, u_{1}\right)$, whereas their degree is 2 in $H_{1}$ and $G_{1}$. Hence, they have degree $q+1=r$ in $B_{q}^{*}\left(S, T, u_{0}, u_{1}\right)$. Finally, the vertices in $V_{1}^{\prime}$ have degree $q$ in $B_{q}\left(S, T, u_{0}, u_{1}\right)$, while they have degree $q-3$ in $H_{1}$ and $G_{1}$. Hence, they have degree $q+q-3=2 q-3=2(r-1)-3=2 r-5$ in $B_{q}^{*}\left(S, T, u_{0}, u_{1}\right)$.

The graph $B_{q}^{*}\left(S, T, u_{0}, u_{1}\right)$ has girth at least 5 , since we are under the hypothesis of Theorem 4.9. In fact, $\Omega\left(H_{0}\right) \cap \Omega\left(H_{1}\right)=\Omega\left(H_{0}\right) \cap \Omega\left(G_{1}\right)=\left\{\frac{q+3}{2}\right\}$, but $M_{H_{0}} \cap M_{H_{1}}=$ $M_{H_{0}} \cap M_{G_{1}}=\emptyset$, since the only edge of weight $\frac{q+3}{2}$ in $H_{0}$ is $\left(\frac{3 q+3}{4}, \frac{q-3}{4}\right)$, while the edges $\left(0, \frac{q-3}{2}\right)\left(0, \frac{q+3}{2}\right)$ are those of weight $\pm \frac{q+3}{2}$ in $H_{1}$ and $G_{1}$. We also have that $\Omega\left(G_{0}\right) \cap \Omega\left(H_{1}\right)=$ $\Omega\left(G_{0}\right) \cap \Omega\left(G_{1}\right)=\emptyset$, which implies that $M_{G_{0}} \cap M_{H_{1}}=M_{G_{0}} \cap M_{G_{1}}=\emptyset$. Moreover, the girth is exactly five, since the 5 -cycle $\left((0,0)_{0},\left(0, \frac{q+1}{4}\right)_{0},\left(0, \frac{q-1}{2}\right)_{0},\left(0, \frac{q-1}{2}\right)_{1},(0,0)_{1}\right)$ lies in $B_{q}^{*}\left(S, T, u_{0}, u_{1}\right)$.

Finally, the order of $B_{q}^{*}\left(S, T, u_{0}, u_{1}\right)$ is $2 q^{2}-q\left(u_{0}+u_{1}\right)-|S|-|T|=2 q^{2}-q-2=$ $r(2 r-5)+1$, by Lemma 4.7 , which satisfies exactly Down's bound (1)

$$
n(\{r, 2 r-5\} ; 5)=1+\sum_{i=1}^{2}(2 r-5)(r-1)^{i-1}=r(2 r-5)+1 .
$$

Hence, the graph $B_{q}^{*}(S, T, 0,1)$ is an $(\{r, 2 r-5\} ; 5)$-cage.

\subsection{Construction for primes $q=4 n+1, n \geqslant 3$.}

Let $B_{q}^{*}\left(S, T, u_{0}, u_{1}\right)$ be the graph resulting from the following choice of its parameters:

\begin{tabular}{|l|c|c|l|}
\hline \multicolumn{5}{|c|}{$S=\left\{\frac{q-1}{4},-\frac{q-1}{4}\right\}=\left\{\frac{q-1}{4}, \frac{3 q+1}{4}\right\} ; \quad T=\emptyset ; \quad u_{0}=0 ;$} & $u_{1}=1$ \\
\hline Graph & Vertices & Edges & Description \\
\hline$H_{0}$ & $\mathbb{Z}_{q}-\left\{\frac{q-1}{4}, \frac{3 q+1}{4}\right\}$ & $\left\{\left(j, j+\frac{q-1}{2}\right) \mid j \in \mathbb{Z}_{q}-\left\{\frac{q-1}{4}, \frac{3 q+1}{4}, \frac{3 q-3}{4}\right\}\right\} \cup\left\{\left(\frac{3 q-3}{4}, \frac{q+3}{4}\right)\right\}$ & $\begin{array}{l}(q-2) \text {-cycle } \\
\text { sums modulo } q\end{array}$ \\
\hline$G_{0}$ & $\mathbb{Z}_{q}$ & $\begin{array}{c}\left\{\left(j, j+\frac{q-1}{2}\right) \mid j \in\left\{\frac{q-1}{2}, \frac{q+3}{2}\right\}\right. \\
\text { sums modulo } q\end{array}$ & $\begin{array}{l}q-\text { cycle } \\
\Omega\left(G_{0}\right)=\left\{\frac{q-1}{2}\right\}\end{array}$ \\
\hline$H_{1} \cong G_{1}$ & $\mathbb{Z}_{q}$ & $\left.\left\{(0, j): j \in \mathbb{Z}_{q}^{*}-\left\{\frac{q-1}{2}, \frac{q+1}{2}\right\}\right\} \cup\left\{\left(\frac{q-1}{4}, \frac{q-1}{2}\right),\left(\frac{3 q+1}{4}, \frac{q+1}{2}\right)\right\}\right\}$ & $\Omega\left(H_{1}\right)=\Omega\left(G_{1}\right)=\mathbb{Z}_{q}^{*}-\left\{\frac{q-1}{2}\right\}$ \\
\hline
\end{tabular}

Theorem 5.11. Let $q=4 n+1$ be a prime, for $n \geqslant 3$. Let $S, T, u_{0}, u_{1}, H_{0}, H_{1}, G_{0}$ and $G_{1}$ be defined as above. Then the amalgam graph $B_{q}^{*}\left(S, T, u_{0}, u_{1}\right)$ is an $(\{r, 2 r-5\} ; 5)-$ cage of order $r(2 r-5)+1$, where $r=q+1$.

Proof. Analogous to the proof of Theorem 5.10. 


\section{New semiregular $(\{r, r+1\} ; 5)$-cages for $r=5,6$.}

In this section we construct two new semiregular cages, namely a $(\{5,6\} ; 5)$-cage with 31 vertices and a $(\{6,7\} ; 5)$-cage with 43 vertices. For the first one, we choose $S, T, u_{0}, u_{1}, H_{0}$, $H_{1}, G_{0}$ and $G_{1}$ to construct the amalgam graph $B_{q}^{*}\left(S, T, u_{0}, u_{1}\right)$ and the result is obtained as a consequence of Theorem 4.9 and Lemma 4.8. The second one is a sporadic example in which we adapt and slightly generalize the techniques that we have used so far.

\subsection{Construction of the $(\{5,6\} ; 5)$-cage.}

Let $G F(4)=\left\{0,1, \alpha, \alpha^{2}\right\}$ be the finite field of order 4 and let $B_{q}^{*}\left(S, T, u_{0}, u_{1}\right)$ be the graph resulting from the following choice of its parameters (c.f. Figure 2):

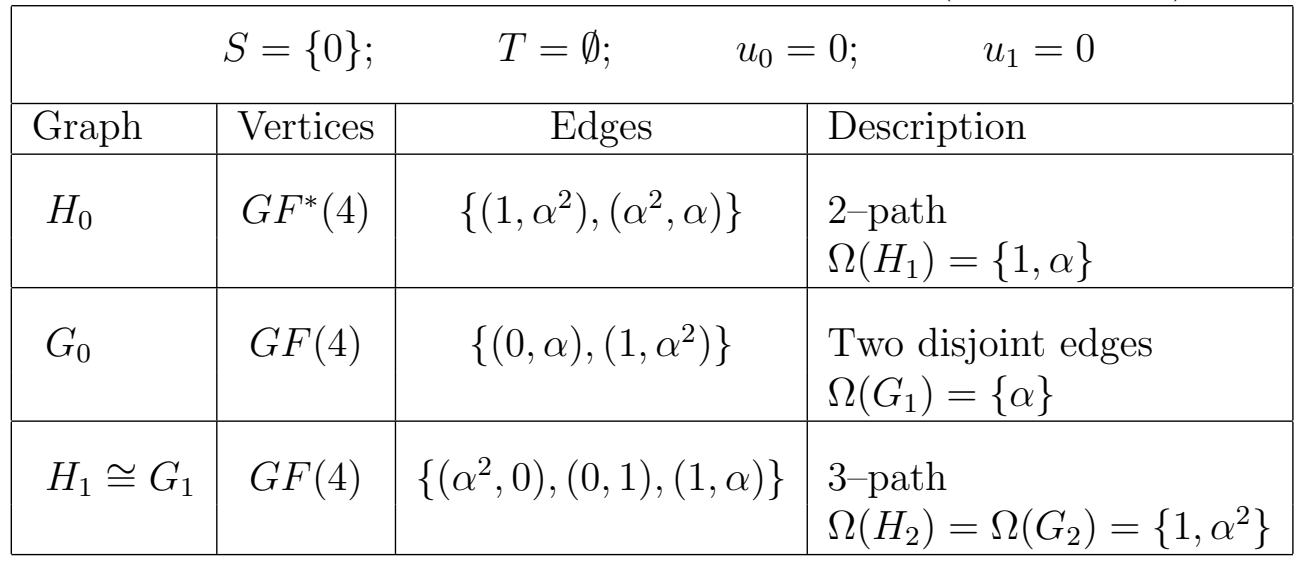

Theorem 6.12. Let $q=4$ and let $S, T, u_{0}, u_{1}, H_{0}, H_{1}, G_{0}$ and $G_{1}$ be defined as above. Then the amalgam graph $B_{4}^{*}\left(S, T, u_{0}, u_{1}\right)$ is a $(\{5,6\} ; 5)$-cage of order 31 .

Proof. From Lemma 4.8 we have that the degree set $D\left(B_{4}^{*}\left(S, T, u_{0}, u_{1}\right)\right)=\{5,6\}$, and moreover, the set of vertices of degree 6 is $\left\{(i, 1)_{0}: i \in G F(4)\right\} \cup\left\{\left(0, \alpha^{2}\right)_{1}\right\}$.

The graph $B_{4}^{*}\left(S, T, u_{0}, u_{1}\right)$ has girth at least 5 , since we are under the hypothesis of Theorem 4.9. In fact, $\Omega\left(H_{0}\right) \cap \Omega\left(H_{1}\right)=\Omega\left(H_{0}\right) \cap \Omega\left(G_{1}\right)=\{1\}$, but $M_{H_{0}} \cap M_{H_{1}}=$ $M_{H_{0}} \cap M_{G_{1}}=\emptyset$, since $\left(\alpha^{2}, \alpha\right)$ is the only edge of weight 1 in $H_{0}$, while the only edges of such weight is $(0,1)$ in $H_{1}$ and $G_{1}$. We also have that $\Omega\left(G_{0}\right) \cap \Omega\left(H_{1}\right)=\Omega\left(G_{0}\right) \cap \Omega\left(G_{1}\right)=\emptyset$, which implies that $M_{G_{0}} \cap M_{H_{1}}=M_{G_{0}} \cap M_{G_{1}}=\emptyset$. Moreover, the girth is exactly five, since the 5 -cycle $\left((0,0)_{0},(0,1)_{0},(0, \alpha)_{0},(1, \alpha)_{1},(1,0)_{1}\right)$ lies in $B_{4}^{*}\left(S, T, u_{0}, u_{1}\right)$.

Finally, by Lemma 4.7 we have that the order of the graph is $2 q^{2}-q\left(u_{0}+u_{1}\right)-|S|-|T|=$ $2(16)-1=31$.

\subsection{Construction of the $(\{6,7\} ; 5)$-cage.}

For this construction we need to modify Reduction 1 and apply the amalgam operation accordingly as follows: 


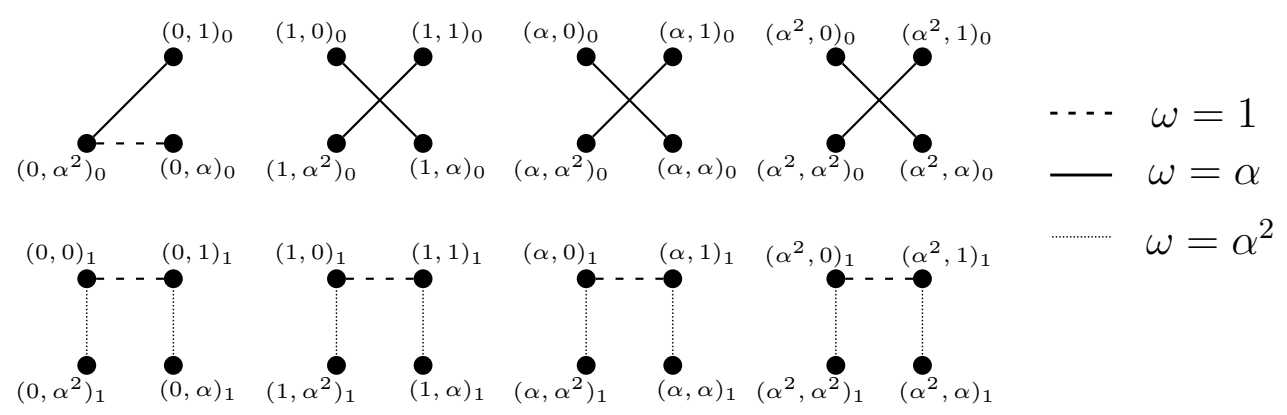

Figure 2: $B_{4}^{*}(S, T, 0,0)-E\left(B_{4}\right)$ with $S=\{0\}$ and $T=\emptyset$

Let $q=5$ and consider the graph $B_{5}$. We modify Reduction 1 removing vertices from $P_{0}$ and vertices of $V_{1}$ from each block $L_{j}$ for $j \in G F(5)$ (whereas in Reduction 1 we delete vertices of $V_{1}$ only from the block $\left.L_{0}\right)$.

Let $T:=\{3\} \subset G F(5), S:=\{0,3\} \subset G F(5), S_{0}=\left\{(0, y)_{0} \mid y \in S\right\} \subseteq P_{0}, T_{j}=$ $\left\{(j, b)_{1} \mid b \in T\right\} \subseteq L_{j}$ for $j \in G F(5)$, and let $B_{5}(S, T T):=B_{5}-S_{0}-\bigcup_{j \in G F(5)} T_{j}$. Note that the graph $B_{5}(S, T T)$ has degree set $D\left(B_{5}(S, T T)\right)=\{q-1, q\}=\{4,5\}$ and order $2 q^{2}-|S|-q|T|=50-2-5=43$. Moreover, each vertex in $V_{0}-P_{0}$ has degree $q-|T|=4$, each vertex of $V_{1}$ with second coordinate in $S-T$ has degree $q-|S-T|=4$, and all other vertices have degree $q=5$.

Now we will amalgam some graphs into $B_{5}(S, T T)$. Let $H_{0}:=\{(2,4),(4,1)\}, G_{0}:=$ $\{(0,2),(2,4),(4,1),(1,3),(3,0)\}$ and $H_{1}:=\{(4,0),(0,1),(1,2)\}$. These graphs are a $2-$ path, a 5-cycle and a 3-path, respectively, with weights $\Omega\left(H_{0}\right)=\Omega\left(G_{0}\right)=\{2\}$ and $\Omega\left(H_{1}\right)=\{1\}$.

Let $B_{5}^{*}(S, T T)$ be the graph obtained from the amalgam of $H_{0}$ into $P_{0}^{\prime}:=P_{0}-S_{0}, G_{0}$ into $P_{i}$, for all $i \in G F^{*}(5)$, and $H_{1}$ into $L_{j}^{\prime}=L_{j}-T_{j}$, for all $j \in G F(5)$ (c.f. Figure 3 for an illustration).

Theorem 6.13. Let $q=5$ and let $S, T, H_{0}, H_{1}$ and $G_{0}$ be defined as above. Then the amalgam graph $B_{5}^{*}(S, T T)$ is a $(\{6,7\} ; 5)$-cage of order 43 .

Proof. Using the same reasoning as in the proof of Theorem 4.9, the amalgam graph $B_{5}^{*}(S, T T)$ has girth at least 5 , since $\Omega\left(H_{0}\right) \cap \Omega\left(H_{1}\right)=\Omega\left(G_{0}\right) \cap \Omega\left(H_{1}\right)=\emptyset$. The girth is exactly 5 , since $G_{0}$ is a 5 -cycle. Moreover, the degree set $D\left(B_{5}^{*}(S, T T)\right)=\{6,7\}$, since all vertices of $B_{5}(S, T T)$ of degree 4 obtain two new edges in $B_{5}^{*}(S, T T)$, and similarly the vertices of $B_{5}(S, T T)$ of degree 5 obtain one or two new edges in $B_{5}^{*}(S, T T)$. Hence, $B_{5}^{*}(S, T T)$ is a $(\{6,7\} ; 5)$-cage as desired, since its order satisfies Down's bound.

There is a further and simpler way of constructing this graph: let $G$ be the HoffmanSingleton Graph [26], consider a vertex $x \in V(G)$ and $N(x)=\left\{x_{1}, x_{2}, \ldots, x_{7}\right\}$ its set 

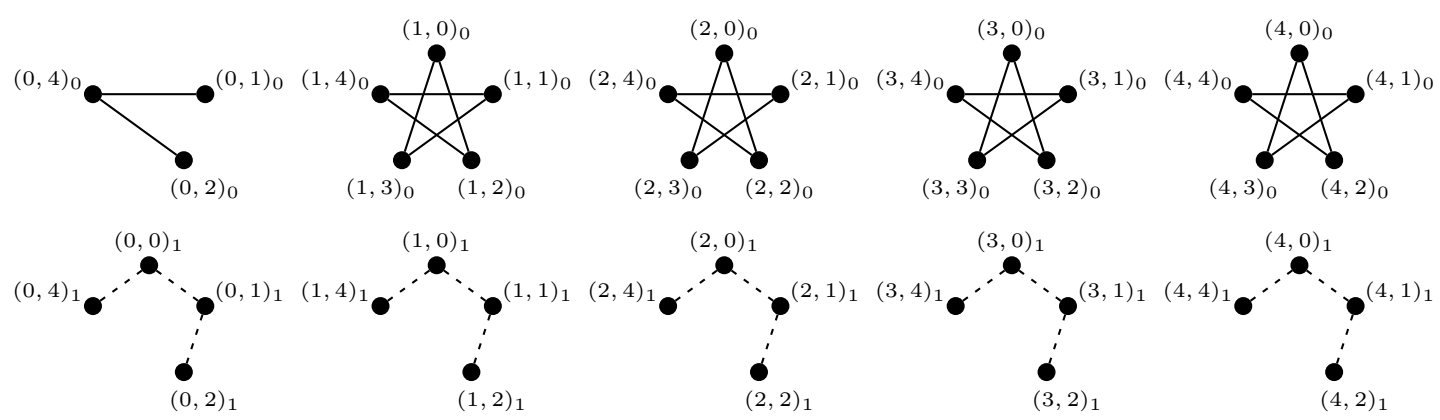

Figure $3: B_{5}^{*}(S, T T)-E\left(B_{5}\right)$ with $S=\{0,3\}$ and $T=\{3\}$

of neighbors, then the graph $H$ obtained from $G$ by deleting the set of vertices $\{x\} \cup$ $\left(N(x)-x_{1}\right)$ is clearly a graph of girth 5 and 43 vertices with 6 vertices of degree 7 and the rest of degree 6 . The graph $H$ is a $(\{6,7\}) ; 5)$-cage as desired, since its order satisfies Down's bound. It is not difficult to prove that this graph $H$ is isomorphic to the previous $B_{5}^{*}(S, T T)$.

It is widely known that Moore graphs of odd girth are very rare: complete graphs, the Petersen graph, the Hoffman-Singleton Graph and maybe the (57,5)-cage. Therefore, the deletion technique carried out for the Hoffman-Singleton Graph can be only applied for this case and may be for $r=57$.

\section{References}

[1] M. Abreu, G. Araujo-Pardo, C. Balbuena, D. Labbate. Families of Small Regular Graphs of Girth 5. Discrete Math. 312(18) (2012) 2832-2842 doi:10.1016/j.disc.2012.05.020

[2] M. Abreu, C. Balbuena, D. Labbate. Adjacency matrices of polarity graphs and other $C_{4}$-free graphs of large size Des. Codes Cryptogr. 55(2-3) (2010) 221 - 233.

[3] M. Abreu, M. Funk, D. Labbate, V. Napolitano. On (minimal) regular graphs of girth 6. Australas. J. Combin. 35 (2006) 119-132.

[4] M. Abreu, M. Funk, D. Labbate, V. Napolitano. A family of regular graphs of girth 5. Discrete Math. 308(10) (2008) 1810-1815.

[5] M. Abreu, M. Funk, D. Labbate, V. Napolitano. A $(0,1)$-matrix framework for elliptic semiplanes. Ars Combinatoria 88 (2008) 175-191.

[6] G. Araujo-Pardo, C. Balbuena, P. García-Vázquez, X. Marcote, J.C. Valenzuela, On the order of $(\{r, m\} ; g)$-cages of even girth, Discrete Math. 308 (2008) 2484-2491.

[7] G. Araujo-Pardo, C. Balbuena, J.C. Valenzuela, Constructions of bi-regular cages, Discrete Math. 309 (2009) 1409-1416.

[8] G.Araujo-Pardo, C. Balbuena, T. Héger, Finding small regular graphs of girth 6, 8 and 12 as subgraphs of cages, Discrete Math. 310 (2010) 1301-1306.

[9] G. Araujo-Pardo, C. Balbuena, Constructions of small regular bipartite graphs of girth 6 . Networks 57(2) (2011) 121-127. 
[10] G. Araujo-Pardo, C. Balbuena, G. López-Chávez, L. Montejano, Bi-regular small graphs of even girth at least 8 . Submitted.

[11] G. Araujo-Pardo, D. González-Moreno, J.J. Montellano, O. Serra. On upper bounds and conectivity of cages, Australas J. Combin. 38 (2007), 221-228.

[12] C. Balbuena, Incidence matrices of projective planes and other bipartite graphs of few vertices, Siam Journal of Discrete Math. 22(4) (2008) 1351-1363.

[13] N. Biggs, Algebraic Graph Theory, 2n edition., Cambridge Univ. Press, Cambridge, 1993.

[14] N. Biggs, Construction for cubic graphs with large girth, Electron. J. Combin. 5 (1998) $56-62$.

[15] J.A. Bondy and U.S.R. Murty, U. S. R. Graph Theory, Springer Series: Graduate Texts in Mathematics, Vol. 244, 2008.

[16] G. Chartrand, L. Lesniak Graphs and Digraphs, Chapman and Hall, $3^{\text {rd }}$ edition, 1996.

[17] G. Chartrand, R.J. Gould, S.F. Kapoor, Graphs with prescribed degree set and girth, Period. Math. Hungar., 6 (1981) 261-266.

[18] M. Downs, R.J. Gould, J. Mitchem, F. Saba, ( $D ; n)$-cages, Congr. Numer. 32 (1981) 179193.

[19] P. Dembowski, Finite Geometries. Springer, New York 1968, reprint 1997.

[20] P. Erdős, H. Sachs H. Regulare Graphen gegebener Taillenweite mit minimaler Knotenzahl, Wiss. Z. Martin- Luther-Univ. Halle-Wittwnberg Math-Naturwiss. Reih. 12 (1963) 251-258.

[21] G. Exoo and R. Jajcay, Dynamic Cage Survey, Electron. J. Combin. 15 (2008) \#DS16.

[22] M. Funk, Girth 5 graphs from elliptic semiplanes, Note di Matematica, 29 suppl. 1 (2009) 91-114.

[23] Z. Füredi, F. Lazebnik, À. Seress, V.A. Ustimenko, and A.J. Woldar, Graphs of prescribed girth and bi-degree, J. Combin. Theory Series B 64(2) (1995) 228-239.

[24] D. Hanson, P. Wang, L. Jorgensen, On cages with given degree sets, Discrete Math., 101 (1992) 109-114.

[25] P. Hafner, Geometric realisation of the graphs of McKay-Miller-Širán̆, J. Combin. Theory Series B 90 (2004) 223-232.

[26] A. J. Hoffman, R. R. Singleton, On Moore graphs with diameters 2 and 3, IBM Journal, November 1960, 497-504.

[27] D.A. Holton and J. Sheehan, The Petersen Graph, Cambridge University, 1993.

[28] S.F. Kapoor, A.D. Polimeni, C.E. Wall, Degree sets for graphs, Fund. Math., 95 (1977) 189-194.

[29] L. Jørgensen, Girth 5 graphs from difference sets, Discrete Math. 293 (2005) 177-184.

[30] F. Lazebnik and V.A. Ustimenko, Explicit construction of graphs with an arbitrary large girth and of large size, Discrete Appl. Math. 60 (1995) 275-284.

[31] W. T. Tutte, A family of cubical graphs. Proc. Cambridge Philos. Soc., (1947) 459-474.

[32] P.K. Wong, Cages - A Survey, J. Graph Theory 6 (1982) 1-22.

[33] Y. Yuansheng, W. Liang, The minimum number of vertices with girth 6 and degree set $D=\{r, m\}$, Discrete Math., 269 (2003) 249-258. 